\title{
Assessment of early side effects of radiotherapy in breast cancer patients
}

\author{
Elaf A. Hussein* \\ MBChB \\ Khudair J. Al-Rawaq* DMRT
}

Abstract:

Background: Breast cancer is the commonest cancer in women. In radiotherapy practice, it comprises $25 \%$ of patient caseload. This makes understanding the breast irradiation toxicities of prime importance. Early radiation toxicities occur during treatment and up to six months after treatment finished.

Objectives: assessment of the early side effects of adjuvant external beam radiotherapy (EBRT) in breast cancer patients.

Fac Med Baghdad

Patients and Methods: A cross sectional survey with analytic component conducted on 60 patients treated in the oncology teaching hospital of medical city from January to April 2016.

Received: July, 2016

Accepted:Aug.2016

Results: The most prevalent toxicities were radiation dermatitis, fatigue, pain, sore throat, nausea, dysphagia, and arm edema. There was a significant difference between prevalence of dermatitis with different radiation doses used. Dermatitis was more prevalent with conventional fractionation $(\mathrm{CF})$, than in hypofractionated radiotherapy.

Conclusion: Dermatitis was the most common acute toxicity of radiotherapy in breast cancer patients with prevalence of $81.6 \%$. It was related to dose fractionation. Fatigue was the 2 nd common (70\%). It was correlated with disease stage.

Keywords: Breast cancer, Conventional fractionation, Hypofractionation, Radiation Dermatitis, Fatigue, Sore throat.

Introduction:

Breast cancer is a major public health problem. It is the most frequently diagnosed cancer globally and is the leading cause of cancer-related death in women1. It is the most common cancer in Iraq; represent $34.4 \%$ of all cancers newly diagnosed in females in Iraq2. Radiation therapy plays a critical role in the management of breast cancer. In a general radiation oncology practice, breast cancer typically comprises approximately $25 \%$ of total patient caseload3.

Early stage breast cancer can be treated effectively either by mastectomy or by breast conserving surgery (BCS) followed by radiotherapy which is an increasing attitude nowadays 4 . This may explain the increased use of EBRT. In addition most locally advanced breast cancers need radiation therapy after mastectomy. If level I axillary nodes are involved, irradiation may be given to levels II and III axillary and SCF nodes. When four or more nodes, a single node $>2 \mathrm{~cm}$ or level III nodes are involved, SCF radiotherapy is indicated5.

Radiotherapy is simply the use of ionizing radiation to kill cancer cells or treat some benign tumors6. It has been proven that systemic treatment and radiotherapy are independent factors influencing the outcome of patients with operable breast cancer, and that both improve locoregional control, disease free, and overall survival7. EBRT is delivered by

*Dept. of Radiation oncology, College of Medicine, University of Baghdad.

Elaf_Ali85@yahoo.com using radiation sources from outside of the body. The required dose is divided (fractionated) and delivered daily for a predetermined time, taking into account the differences in repair and repopulation times between tumor cells and normal cells8. In breast cancer, the radiation therapy schedule could be either 40 Gy in 15 daily fractions of 2.67 Gy given in 3 weeks or 42.5 Gy in 16 daily fractions of 2.66 Gy given in 3.5 weeks or $50 \mathrm{~Gy}$ in 25 daily fractions given in 5 weeks5. There has been a growing trend toward hypofractionation, which involves delivering a higher dose per fraction for a shorter number of fractions for a biologically equivalent dose. This has been shown to be safe and effective as a standard treatment schedule in multiple randomized trials3. Standard opposed tangential fields remains the most commonly employed method for delivery of whole-breast irradiation. A number of publications have explored the potential advantages of 3DCRT to treat patients with breast cancer3. Theoretically, 3DCRT involves a reduction in the volume of normal tissues receiving a high dose, with an increase in dose to the target volume.

Radiotherapy works by killing cancer cells that may be present in the breast or chest wall following surgery. However, the normal cells in the area being treated are also affected. These normal tissue cells have a greater ability to recover from the radiation compared to cancer cells. Despite this recovery, side effects do occur and are usually a normal part of treatment and may affect the quality of life. Some side effects occur "early 
(These are side effects that occur during treatment and up to six months after treatment has finished) whilst others may develop "late (several months to years after completing treatment)9. It is very important to study these side effects; however till now knowledge about these topics is limited. Some of the possible early side effects after radiotherapy to the breast are9 common like skin reaction, fatigue, pains in the breast or chest wall area, sore throat and dysphagia; some are uncommon like radiation pneumonitis, lymphedema, Nausea and vomiting while others are rare like heart damage.

Aims of the study: The study aims to find the prevalence and the severity of acute radiation side effects in breast cancer patients, to assess the potential relation of the possible early side effects with the doses fractionation schemes and to assess the potential relation between the possible acute toxicities and the type of surgery _mastectomy versus BCS_ to refine treatment decisions.

\section{Patients and Methods:}

This study has been designed to be a cross sectional survey with analytic components to evaluate the early side effects of breast irradiation in breast cancer patients treated with adjuvant three dimensional conformal radiotherapy (3DCRT) after mastectomy or BCS. The study involved 60 patients with breast cancers of variable stages and histopathology treated with adjuvant 3DCRT in the radiotherapy and nuclear medicine department of the oncology teaching hospital of medical city complex in Baghdad from January to April 2016. The patients were simulated on wide bore 16 slices CT simulator (PHILIPS), planned with Xio software and treated on ELEKTA linear accelerator (synergy and Infinity). The patients who were included in the study had been treated either by CF or by hypofractionation scheme. They were seen 10 days after their first radiation session, during irradiation period, and in the follow up visits 2 months after completing their schedule of radiation. A questionnaires filled by the doctors (after taken permission from the patient) were used in which notes about the side effects, their intensity and other information about the patients were taken by history and physical examination. Clinical data on tumor characteristics and therapeutic regime were abstracted from patient records saved in the radiation center (patients files and from Mosaiq). There were no exclusion criteria or age limits in this study.

The patients had been asked about nine main complaints (fatigue, nausea and/or vomiting, symptoms of radiation dermatitis, Pain in the irradiated area, swelling of the affected breast and/or the ipsilateral arm or difference in the size between arms, sore throat and/or voice changes, dysphagia, cardiac and respiratory symptoms). The patients then had been examined to see if there was any sign of dermatitis or arm edema. Any patient who had cardiac or pulmonary symptoms were offered to do further investigations like CXR, ECG, ECHO if they accepted, the results were also recorded.

Acute Adverse reactions had been graded according to the common terminology criteria for adverse events (CTCAE) version 4.0 published on June 14, 2010 By U.S. department of health and human services.10 See table 1. Maximum toxicity during the study period had been depended.

To start the results analysis, our sample then were classified according to their type of surgery (mastectomy versus BCS), the fractionation of the dose $(5000,4005,4260 \mathrm{cGy})$ and also divided into those who received supraclavicular irradiation and those who had only breast or chest wall irradiation. Differences in the prevalence of adverse effects between these variants were studied. Differences in the side effects across different age groups, different cancer stages, comorbidities, previous chemotherapeutic regimens and different intervals between radiation and the last cycle of chemotherapy were also assessed. Univariate statistical analyses were done using IBM SPSS Version 22. Chi-square test (or Fisher's exact test), t-test for independent samples was used for statistical analyses, as appropriate. A significance level of 5\% was chosen.

Table1: CTCAE grading of adverse events

\begin{tabular}{ccccc}
\hline Adverse Event & Grade 1 & Grade 2 & Grade 3 & Grade 4 \\
$\begin{array}{c}\text { Radiation } \\
\text { dermatitis }\end{array}$ & $\begin{array}{c}\text { faint erythema } \\
\text { or dry } \\
\text { desquamation }\end{array}$ & $\begin{array}{c}\text { moderate to brisk erythema, } \\
\text { Patchy moist desquamation, } \\
\text { mostly confined to skin folds } \\
\text { and creases, moderate edema }\end{array}$ & $\begin{array}{c}\text { moist desquamation other } \\
\text { than skin folds and creases, } \\
\text { bleeding induced by minor } \\
\text { trauma or abrasion }\end{array}$ & $\begin{array}{c}\text { skin necrosis or } \\
\text { ulceration of full } \\
\text { thickness dermis, } \\
\text { spontaneous } \\
\text { bleeding }\end{array}$ \\
\hline Fatigue & $\begin{array}{c}\text { Fatigue } \\
\text { relieved by rest }\end{array}$ & $\begin{array}{c}\text { Fatigue not relieved by rest; } \\
\text { limiting instrumental ADL }\end{array}$ & $\begin{array}{c}\text { Fatigue not relieved by } \\
\text { rest, limiting self-care ADL }\end{array}$ & $\ldots . .$. \\
\hline $\begin{array}{c}\text { Pain breast pain, } \\
\text { chest wall pain }\end{array}$ & Mild pain & $\begin{array}{c}\text { Moderate pain; limiting } \\
\text { instrumental ADL }\end{array}$ & $\begin{array}{c}\text { Severe pain; limiting self- } \\
\text { care ADL }\end{array}$ & $\ldots . . . .$. \\
\hline
\end{tabular}

ADL: Activities of Daily Living 


\section{Results:}

The study involves 59 females and only one male. Their ages range from 27 to 71 years with a mean age of 49 years. Most of the patients were treated with mastectomy $(73.3 \%)$, while $26.7 \%$ were treated with BCS. Right sided breast cancers were slightly more common than left breast cancers $(56.7 \%)$. There was only one case of synchronous bilateral breast cancer. The most common site of the tumor was the upper outer quadrant (55.2 \%). Most of the patient presented with advanced stage,
$40.7 \%$ of cases presented with stage IIIa, and $27.1 \%$ were stage IIIc Patients. All the patients were treated in a supine position on breast board. Only 4 cases received booster doses in which wedges were used; these cases were excluded from calculations related to the dose. There were 3 dose fractionations used in this study: CF; $5000 \mathrm{cGy} / 25$ Fraction (20\%), hypofractionation were to give $4260 \mathrm{cGy} / 16$ Fraction $(21.7 \%$ of cases) or 4005 cGy/15 Fraction (58.3\%). The distribution of early side effects can be summarized by figure1.

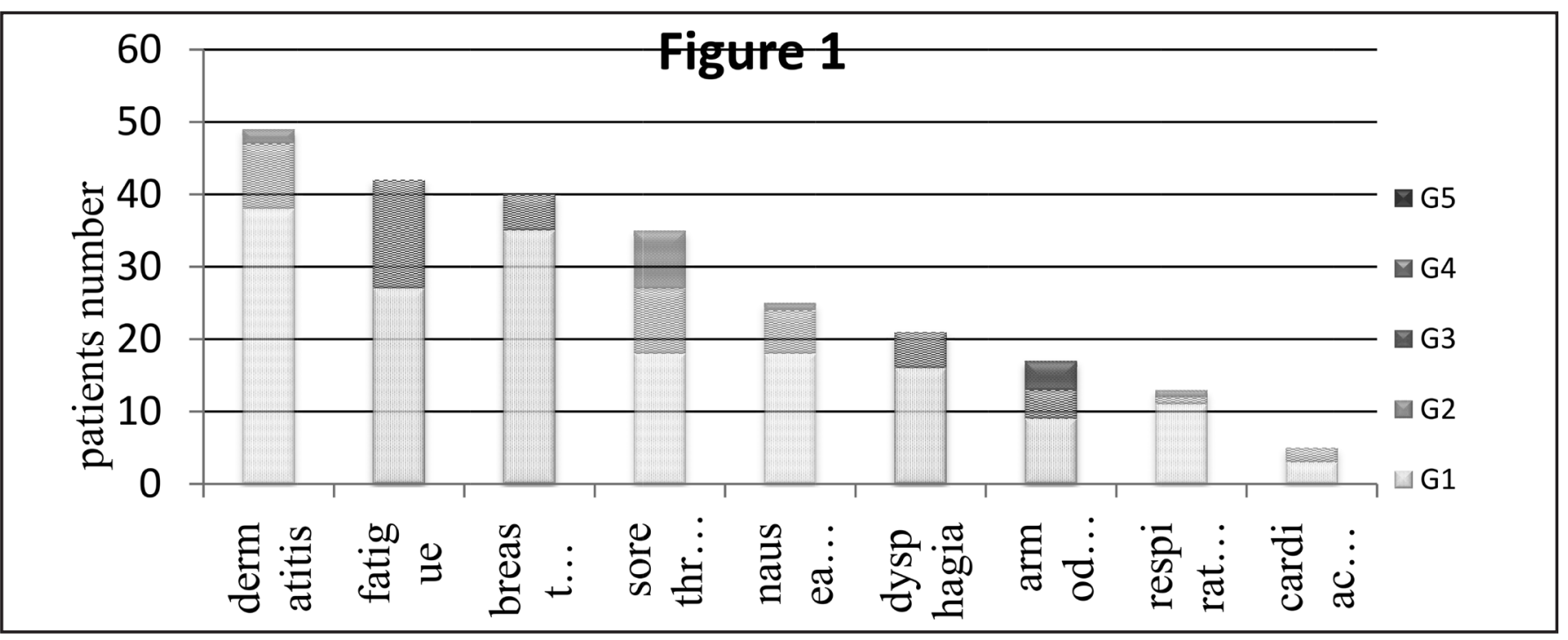

Figure 1: Summery of early side effects of radiotherapy in breast cancer patients

Most of the skin reactions had been developed after a mean of 11.8 days after start of radiation. Figure 2 compare the time latency of three adverse events.

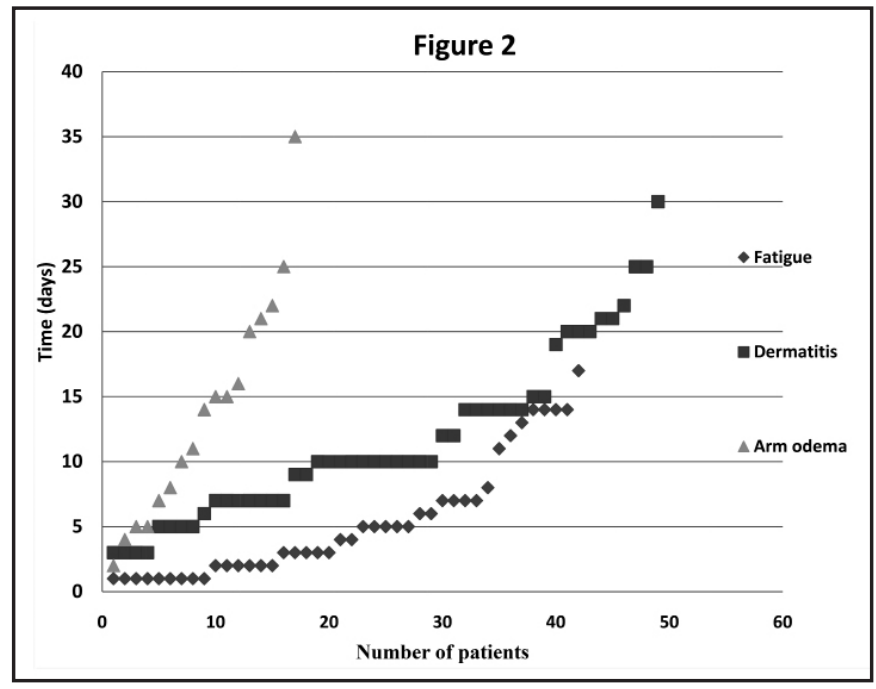

Figure 2: Comparison of time to develop dermatitis, fatigue and arm edema

Dermatitis was the most common side effect in this study. The overall prevalence of dermatitis is $81.6 \%$. There was a significant difference between prevalence of dermatitis among the 3 groups of fractionation, see table 2. There was also more sever dermatitis i.e. high grade (Grade 2 and 3) among those who receive $5000 \mathrm{cGy}$ (prevalence of $41.7 \%$ ), and those who receive $4260 \mathrm{cGy}(23.1 \%)$ while only $(9.7 \%)$ of patients treated by 4005 cGy develop higher than grade 1 dermatitis (At $\mathrm{P}$ 0.049). The risk of developing higher than grade 1 dermatitis in conventional radiation is about 5 times the risk in hypofractionation treatment (P 0.045 using fisher's exact test). The prevalence of dermatitis was also more in patient treated with BCS (87.5\%) than those who had mastectomy (79.5\%); however, the relation was not significant. (P 0.71)

Table 2: The relation of dose with dermatitis prevalence

\begin{tabular}{cccc}
\hline & \multicolumn{2}{c}{ Dermatitis prevalence } & \multirow{2}{*}{ Total } \\
\cline { 2 - 3 } & Dermatitis & No dermatitis & \\
\hline 4005 & $21(67.7 \%)$ & $10(32 \%)$ & $31(100 \%)$ \\
\hline 4260 & $12(92.3 \%)$ & $1(7.7 \%)$ & $13(100 \%)$ \\
\hline 5000 & $12(100 \%)$ & $0(0 \%)$ & $12(100 \%)$ \\
\hline Total & $45(80.4 \%)$ & $11(19.6 \%)$ & $56(100 \%)$ \\
\hline
\end{tabular}

P value 0.028 according to fisher's exact test

Fatigue was the 2nd common early side effect (70\%). There was no statistical significant difference in the Fatigue prevalence according to type of surgery or radiation dose used. Also there was no significant difference between the mean 
ages of patients who had fatigue (49 year) and of those who didn't have any (48.9 year). There were no significant relations with other comorbidities or with the chemotherapy type. There was positive correlation between cancer stages and fatigue ( $\mathrm{P}$ 0.03 ). Pain at the scar and the breast is also prevalent, $66.7 \%$ and more common in patients treated with $4260 \mathrm{cGy}$ while less prevalent in patient treated with 4005 cGy, p 0.24.

Most of the patients were lymph nodes positive, $88.3 \%$. Most of patients received SCF irradiation 90\% (54 cases) and only 6 patients had chest wall or breast irradiation only, the prevalence of sore throat in those 6 patients is only $33.3 \%$, while it was $61.1 \%$ in those who received SCF irradiation. 3 patients also developed voice changes. However, no significant correlation between lymph node status and sore throat severity was found. Seventy two percent of nausea cases were mild (grade 1), few cases were associated with vomiting.

The entire patient had surgical axillary lymph node sampling; no one had SLN sampling. Of the seventeen cases with breast and arm edema in this study, 6 cases already had edema before radiation but they relapsed or aggravated after radiation, all of them were above grade $1(50 \% \mathrm{G} 2$ and $50 \% \mathrm{G} 3)$. In the other hand most cases that develop edema after radiation (11 cases) were mild (G1 81.8\%). There were no significant correlations between arm edema prevalence and lymph node status.

Only two patients developed high grade (G2, G3) respiratory symptoms. And only one of the thirteen patients who developed respiratory symptoms had positive chest x-ray findings. Only five patients had mild to moderate cardiac symptoms, four of them refused to do further investigations and one had normal investigations.

\section{Discussion:}

Radiotherapy in breast cancer patients is relatively well tolerable. Using modern-day technology, using computed tomography (CT) simulators, modern-day linear accelerators, computerized treatment planning modalities, and on-board imaging techniques, the therapeutic ratio has markedly improved, whereas the potential for side effects has diminished significantly 11. No patient in this study developed severe toxic events that necessitate interruption of treatment; however, every patient had developed at least three or more toxicities after their radiation. Radiation Dermatitis was the most prevalent acute side effect, this is consistent with literature 12. The overall prevalence of dermatitis was $81.6 \%$ which was not so far from the radiation acute skin reaction incidence in many other researches, for example; Podrock D and Kristjanson $\mathrm{L}$ found that Over $90 \%$ of women who receive radiation for breast cancer will as a result develop some skin changes during their course of treatment12. Most skin reactions were mild erythema and dryness of the skin in the irradiated area (CTCAE G1 reaction), only 15\% developed G2 and $3.3 \%$ developed G3 dermatitis in this study, no one develop skin necrosis or ulceration (G4 reaction). This doesn't go with most of the previous researches except Sharp et al work. De Langhe et al, in his study stated that $58 \%$ of patients developed G2+ dermatitis, but we should notice that in this study IMRT was used, this may explain why skin received higher doses13.

The skin reactions had been developed after a mean of 11.8 days after radiation start. This goes with most of previous data in the literature review. "Skin reactions related to radiotherapy usually manifest within 1-4 weeks of radiation start" 21 when damaged basal cells migrate to the skin surface14.

Hypofractionation (especially $4005 \mathrm{cGy} / 15 \mathrm{~F}$ ) was seen to be protective against skin reaction (P 0.028). Dermatitis was more prevalent in patient receiving CF $(100 \%)$. See table 1 . This is compatible with the rules of radiobiology and to De Langhe work: patients treated with hypofractionated radiotherapy develop less dermatitis when compared to patients treated in the normofractionated regimen $(\mathrm{p}<0.001) 13,15$. Hypofractionated schedules have the additional advantage of being more convenient for the patient and they help to spare resources 25 . There was no significant effect of surgery type on dermatitis. Same findings were seen by Hopwood $\mathrm{P}$ who stated that surgery type had no effect on skin reaction16. Fatigue was the 2nd most common early radiation side effect with a prevalence of $70 \%$. Only $25 \%$ developed CTCAE G2 fatigue and no one developed higher grade fatigue. This is slightly lower than the fatigue incidence in the literature. Escalante results regarding prevalence, propose that between $60-96 \%$ of all patients with cancer, in general, report fatigue. Nearly $80 \%$ of the patients undergoing radiotherapy mention this symptom 17 , and $44 \%$ of them present it in a significant manner, often associated with the previous chemotherapy treatment. Despite the fact that fatigue is a common symptom of breast cancer treatment, we are unaware of any research that has directly compared the characteristics and course of fatigue related to radiotherapy during the active treatment period. There was no statistical significant difference in the Fatigue prevalence according to type of surgery, radiation dose used, and other comorbidities. This is compatible with most of the literature. Donovan K said that there were no significant relationships between fatigue and age, disease stage, and type of surgery 18 But in our study we found positive correlation between the stage of the cancer and fatigue this also contradict with Lavanitit al who found that disease staging did not influence the level of fatigue in their study19 although the authors of that study consider these data inconsistent due to the reduced size of the sample. Proinflammatory cytokines have been cited as the most likely underlying biological mechanism20. Previous research has demonstrated significant relationships between emotional distress and fatigue18. Thus, it may be the case that certain psychological factors, though not the focus of our study, may have accounted for these differences in fatigue at baseline. Also there was no significant difference between the mean ages of 
patients who had fatigue during or after radiation and the mean age of those who didn't have any fatigue; however distribution of fatigue prevalence did change in each age group. It appears as that there was 2 peaks, the first in the age 26-35 and the 2nd above 66 yrs. Breast Pain at the site of surgery, scars, chest wall, breast, nodal region and skin in the irradiated area was also prevalent, $66.7 \%$; this is constituent with Hopwood $\mathrm{P}$ who stated that almost two thirds of patients reported pain in the breast area16. It was more common in patients treated with $4260 \mathrm{cGy}$ while less prevalent in patient treated with $4005 \mathrm{cGy}$, but no statistical significant correlations found. $87.5 \%$ of cases with pain have only mild pain. Nearby results were found in literatures. Sore throat, nausea, vomiting and dysphagia: Sore throat, voice changes and dysphagia usually results from high doses of radiation received by midline structures including larynx, pharynx, esophagus and other mucosal surfaces, so it was hypothesized that these effects are more among those who receive SCF irradiation since the SCF usually treated by opposing anterior-posterior beams. However this hypothesis couldn't be tested in this study because most of the patients treated had advanced stage with positive lymph nodes so they need SCF irradiation (90\%) and only 6 patients had chest wall or breast irradiation. The prevalence of sore throat was less in those 6 patients $(33.3 \%)$, comparing to $61.1 \%$ in those who receive SCF irradiation. Dysphagia may be a result of sore throat rather than esophagitis. Nausea was prevalent in $41.6 \%$ which is more than the prevalence in literature. Risk factors for radiation-induced emesis 21 include previous chemotherapy, radiation of the upper abdomen, and radiation fields greater than $400 \mathrm{~cm} 2$. Arm edema: Of the 17 cases with ipsilateral limb edema included in this study, 6 cases had already edema before radiation but their condition either relapsed or aggravated after radiation, all of them were above grade 1 in contrast to edema after radiation which was mild mostly. There were no significant correlations between arm edema prevalence and lymph node status. Again this may be explained by sample size and advanced stage dominance (Stage III). Only two patients developed high grade (G2 and 3 ) respiratory symptoms. And only one of the 13 patients who developed respiratory symptoms had positive chest x-ray findings. Both the short duration (7.7 days) between start of radiation and symptoms and the negative $x$-ray finding indicate that these symptoms may be not related to radiation; however acute radiation pneumonitis couldn't be excluded by absence of symptoms or radiological findings. The incidence of pulmonary toxicity after radiation therapy for breast cancer varies significantly in the literature but is typically well under $10 \%$ with modern treatment units and techniques. The acute and long-term effects are similar in that they are primarily dependent on the volume of lung irradiated and thus higher with the addition of SCF or internal mammary fields22, 23. Only 5 patients had cardiac symptoms, most of them refuse to do further investigations and one had normal investigations. This low prevalence of cardiac events may be because that cardiac toxicity considered a late rather than early adverse effect. This is consistent with Shapiro $\mathrm{C}$ and other literatures, which stated that acute and subacute cardiac complications of radiation therapy for breast cancer, such as pericarditis and cardiac failure, are rare 24.

\section{Conclusions:}

The most prevalent acute toxicities associated with radiotherapy in breast cancer patients were (in descending order): Acute radiation dermatitis, Fatigue, pain within irradiated area, sore throat, nausea, dysphagia and arm edema. Hypofractionated breast radiation (especially $4005 \mathrm{cGy} / 15$ fraction) may be safer than normofractionation in the form of reduction of dermatitis; however, studies on late normal tissue reaction are strongly recommended. It strongly recommended creating a forma to be used in any new radiation center, to document any acute toxicity of radiation. Further studies on radiation new techniques are strongly recommended since there are no studies on these new fields in Iraq.

\section{Author's contribution:}

Dr. Elaf Ali Hussein: student

Dr. Khudair Jasim Al-Rawaq: supervisor

\section{References:}

1. Forouzanfar M, Delossantos A, Lozano R, Alan D, Murray Ch, Naghavi M. Breast and cervical cancer in 187 countries between 1980 and 2010: a systematic analysis. The Lancet 2011; 6736: 61351-61352.

2. Iraqi cancer board. Iraqi cancer registry. Baghdad: $M O H$; 2014.

3. Perez C, Brady L, Halperin E, Wazer D. Principles and practice of radiation oncology. 6th edition. Philadelphia: Lippincott Williams \& Wilkins; 2013.

4. Kelemen JJ 3rd, Poulton T, Swartz MT, et al. Surgical treatment of early-stage breast cancer in the Department of Defense Healthcare System. J Am Coll Surg 2001; 192(3):293297.

5. Barrett A, Dobbs J, Morris ST, Roques T. Practical Radiotherapy Planning. 4th edition. London: Hodder Arnold; 2009.

6. Society of Radiographers, An overview of radiotherapy [internet]. 2014 [cited 4 May 2016]. Available from: http:// tinyurl.com/p2zyp $5 w$

7. Clarke M, Collins R, Darby S et al. Effects of radiotherapy and of differences in the extent of surgery for early breast cancer on local recurrence and 15-year survival: an overview of the randomized trials. Lancet 2005; 366: 2087-2106.

8. Glover D, Harmer V. Radiotherapy induced skin reactions: assessment and management. British Journal of nursing 2014, 
23(4): 28-35.

9. Radiotherapy following mastectomy [internet]. 2016 [cited 11 May 2016]. Available from: http://www.google.iq/url?sa=t $\& r c t=j \& q=\&$ esrc $=s \&$ source $=$ web $\& c d=1 \& v e d=0 a h U K E w j 4$ iMji4tLMAhVKIMAKHap5C24QFgghMAA\&url=http\%3A\% $2 F \% 2 F w w w . s w s l h d . n s w . g o v . a u \% 2 F c a n c e r \% 2 F p d f \% 2 F B r e a s$ $t \% 2 F R T \% 2520$ Post-mastectomy.pdf\&usg $=$ AFQjCNHX2H8n iCDPLUjEMllGwu8qCRjXFA\&sig2=AFqNU1bPq3Xtu9GCT $H P a O w \& b v m=b v .121658157, d . Z G g$

10. CTCAE version 4.03 [internet]. 2010 [cited 11 May 2016]. Available from: http://www.google.iq/url?sa=t\&rct=j\&q= \&esrc $=s \&$ source $=$ web\&cd $=1 \&$ ved $=0$ ahUKEwjl2 euu $79 L M$ AhVnAsAKHaR8C_0QFggaMAA\&url=http $\% 3 \mathrm{~A} \% 2 \mathrm{~F} \% 2 \mathrm{~F}$ evs.nci.nih.gov\%2Fftp1\%2FCTCAE\%2FCTCAE_4.03_201 0-06-14_QuickReference_8.5x11.pdf\&usg=AFQjCNEbEZ0_ ZlAsucvkYbyW-gL_2RPP8A\&sig2 $=C t K S V R E b J O T Y n Y i v v$ B_Bxw\&bvm $=b v .121658157, d . b G g$

11. Moran M, Haffty B. Radiation Techniques and Toxicities for Locally Advanced Breast Cancer. Semin Radiat Oncol 2009; 19: 244-255.

12. Podrock D and Kristjanson L. Skin reactions during radiotherapy for breast cancer: the use and impact of topical agents and dressings. Eur J Cancer Care 1999; 8:143-153.

13. De Langhe S, Mulliez T, Veldeman L et al. Factors modifying the risk for developing acute skin toxicity after whole-breast intensity modulated radiotherapy. BMC Cancer 2014; 14:711.

14. NHS Quality Improvement Scotland. Skincare of patients receiving radiotherapy [Internet]; 2010. [Cited 29 May 2016] Available from: http://tinyurl.com/og3r6p 7

15. Owen J, Ashton A, BLISS et al. Effect of radiotherapy fraction size on tumor control in patients with early-stage breast cancer after local tumor excision: long-term results of a randomized trial, Lancet Oncol. 2006; 7:467-471.

16. Hopwooda P, Havilandb J, Millsb J, Sumob G, Blissb J. The impact of age and clinical factors on quality of life in early breast cancer: An analysis of 2208 women recruited to the UK START Trial (Standardization of Breast Radiotherapy Trial). The Breast 2007; 16: 241-251.

17. Escalante CP. Treatment of cancer related fatigue: an update. Supportive Care in Cancer 2003; 11: 79-83.

18. Donovan K, Jacobsen P, Andrykowski M, et al. Course of Fatigue in Women Receiving Chemotherapy and/or Radiotherapy for Early Stage Breast Cancer. Journal of Pain and Symptom Management 2004; 28(4):373-379.

19. Lavdaniti $M$, Patiraki E, Dafni $U$, Katapodi $M$, Papathanasoglou E\& Sotiropoulou A. Prospective assessment of fatigue and health status in Greek patients with breast cancer undergoing adjuvant radiotherapy. Oncology Nursing Forum 2006; 33: 603-610.

20. Bower JE, Lamkin DM. Inflammation and cancer related fatigue: mechanisms, contributing factors and treatment implications. Brain Beh Imm. 2013; 30(Suppl):S48-S57.

21. Radiation-induced emesis: a prospective observational multicenter Italian trial. The Italian Group for Antiemetic Research in Radiotherapy. Int J Radiat Oncol Biol Phys. 1999; 44(3):619-625.

22. Lind P, Wennberg B, Gagliardi G, et al. Pulmonary complications following different radiotherapy techniques for breast cancer, and the association to irradiated lung volume and dose. Br Cancer Res Treat 2001; 68:199-210.

23. Krengli M, Sacco M, Loi G, et al. Pulmonary changes after radiotherapy for conservative treatment of breast cancer: $A$ prospective study. Int JRadiat Oncol Biol Phys 2008; 70:14601467.

24. Shapiro C, Recht A. Side effects of adjuvant treatment of breast cancer. $N$ Engl J Med 2001; 344(26):1997-2006. Available from: www.nejm.org. 